Somnologie 2015 · 19:4-5

DOI 10.1007/s11818-015-0702-4

Online publiziert: 12. März 2015

(c) Springer-Verlag Berlin Heidelberg 2015

\section{H. Danker-Hopfe}

Kompetenzzentrum Schlafmedizin, CharitéCentrum 15, Charité - Universitätsmedizin Berlin, Campus Benjamin Franklin, Berlin

\section{Mikrostrukturen des Schlafs}

\section{Ausgewählte Aspekte zu schlafrelevanten Frequenzen und Mustern}

Über die Makrostruktur des Schlafs (Schlafstadien und deren Verteilung) hinausgehende Informationen, die aus dem Elektroenzephalogramm (EEG), dem Elektromyogramm (EMG) und dem Electrookulogramm (EOG) entnommen werden können, gewinnen nicht nur im Forschungs-, sondern auch im klinischen Kontext zunehmend an Bedeutung. Bei den im vorliegenden Heft zusammengestellten Beiträgen zu Mikrostrukturen des Schlafs handelt sich um Übersichtsbeiträge (Rodenbeck et al., Raschke und Moser), um Publikationen, in denen die Bedeutung von spezifischen Mikrostrukturen untersucht wird (Parapatics et al. sowie Gruber et al.), um einen mehr grundlagen- bzw. methodisch orientierten Beitrag (Eggert et al.) sowie um einen Beitrag, der sich unter dem Aspekt von Referenzwerten mit alters- und geschlechtsspezifischen Variationen von Mikrostrukturen des „Rapid-eye-movement“(REM)Schlafs beschäftigt (Danker-Hopfe et al.).

Die Erkennung von schlafrelevanten Frequenzen und Mustern wie z. B. KKomplexen, Spindeln, $\delta$-Wellen u. a. ist von essenzieller Bedeutung für die Zuordnung von Schlafstadien und damit eine wesentliche Voraussetzung für gute klinische Praxis („good clinical practice“, GCP) in der Schlafmedizin. Im Jahr 2006 hat die Deutsche Gesellschaft für Schlafforschung und Schlafmedizin e.V. (DGSM) aus der Arbeitsgruppe (AG) Ausbildung heraus eine Task Force eingerichtet. Diese Task Force hat im Rahmen eines Konsensusverfahrens Interpretationshilfen und - wenn nötig - Spezifikationen zur Verbesserung der Erkennung von Elektroenzephalographie(EEG)-Wellenformen und -Mustern erarbeitet, die zur Verbesserung der Reliabilität der Auswertung von Polysomnographien beitragen sollten. Im vorliegenden Heft finden sich im Beitrag von Rodenbeck et al. überarbeitete Spezifikationen zu Verbesserung und Vereinheitlichung der Erkennbarkeit der schlafrelevanten Frequenzen ( $\alpha$-, $\theta$ - und $\delta$-Wellen) sowie Muster (Vertex- und Sägezahnwellen, K-Komplexe und Schlafspindeln). Die Überarbeitung erfolgte unter Berücksichtigung des Manuals der American Academy of Sleep Medicine (AASM) und neuerer Literatur mit dem Ziel, dass es keine Abweichungen und Widersprüche gegenüber dem AASM-Manual in der aktuellen Fassung 2.1 von 2014 gibt.

Ebenfalls von Bedeutung für die $\mathrm{Zu}-$ ordnung von Schlafstadien sind ,arousals“, die allgemein kurzzeitige Aktivierungsvorgänge charakterisieren. Im Beitrag von Raschke werden die verschiedenen Arten von Arousals definiert, insbesondere diejenigen, die im Schlaf vorkommen. Es werden Mechanismen der Genese von Arousals im Schlaf skizziert, Definitionsmerkmale genannt und - soweit bekannt - ihre klinische Bedeutung erläutert. Raschke weist darauf hin, dass EEGArousals eine Schlaffragmentierung widerspiegeln, deren klinische Bedeutung noch unklar ist.

Eine besondere Form von Arousals im Schlaf sind zyklisch alternierende Schwankungen im EEG-Muster, die als „cyclic alternating pattern“ (CAP) bezeichnet werden. Mit diesem Muster befasst sich der Beitrag von Moser. Es werden Klassifikationskriterien beschrieben, Informationen zum neurophysiologischen Hintergrund gegeben und klinische Anwendungsbereiche aufgezeigt.

Mit der Bedeutung von speziellen Mustern beschäftigen sich auch die Beiträge von Gruber et al. und Parapatics et al. in diesem Heft. Anders als die zuvor beschriebenen allgemein gehaltenen Ausführungen zur Bedeutung von Frequenzen und Mustern werden in den Arbeiten von Gruber et al. sowie Parapatics et al. die Ergebnisse eigener Studien vorgestellt. In der Arbeit von Parapatics et al. wird die Bedeutung von K-Komplexen als Indikator der Schlafhomöostase bei 22 Patienten mit obstruktivem Schlafapnoesyndrom (OSAS) sowie 22 alters- und geschlechtsangepassten Kontrollen untersucht. Während bei unbehandelten OSAS-Patienten die Amplituden der K-Komplexe nicht signifikant abnahmen, zeigte sich 3 bis 6 Monate nach Beginn einer „Continuous-positive-airway-pressure"(CPAP)Therapie, ähnlich wie bei den gesunden Kontrollen, eine Reduktion der Amplituden. Die Autoren leiten daraus ab, dass die Amplituden von K-Komplexen bei Gesunden und bei Patienten als Indikatoren für die Schlafhomöostase herangezogen werden können.

Die Arbeit von Gruber et al. reiht sich ein in die zahlreichen Studien zur Bedeutung von Schlafspindeln und langsamen Wellen für die Gedächtniskonsolidierung 
im Schlaf, insbesondere im Hinblick auf das Hippocampus-abhängige deklarative Gedächtnis. In einer früheren Studie an 24 gesunden jungen Probanden konnten die Autoren zeigen, dass über die Nacht auftretende Veränderungen der Abrufleistung in einer Wortpaarassoziationsaufgabe signifikant mit Änderungen der Spindelaktivität zwischen der Lern- und einer Kontrollnacht korrelierten. Die Ergebnisse der aktuellen Studie unterstreichen die Bedeutung der schnellen Schlafspindeln im Stadium 2 für die deklarativen Gedächtnisprozesse. Ferner zeigte sich, dass die Korrelationen der Abrufleistungen mit Schlafspindeln nur für das frühe Stadium S2 zu beobachten ist.

Studien zur Bedeutung von Mikrostrukturen, wie z. B. Schlafspindeln im Zusammenhang mit der Konsolidierung von deklarativen Gedächtnisleistungen, basieren in der Regel auf jeweils einer Kontroll- und einer Untersuchungsnacht pro Experimentalbedingung, wobei intraindividuelle Variationen unberücksichtigt bleiben (müssen). Die Arbeit von Eggert et al. beschäftigt sich daher mit der individuellen Stabilität von Schlafspindelparametern. Untersucht wird diese Stabilität an einer Stichprobe von 32 jungen Männern im Alter von 20 bis 30 Jahren auf der Basis von jeweils 9 Nächten, in denen entsprechende Ableitungen erfolgten. Die Ergebnisse unterstreichen zum einen die Nacht-zu-Nacht-Stabilität und zum anderen, dass die Variabilität innerhalb von Individuen deutlich geringer ausgeprägt ist als die zwischen verschiedenen Personen.

Diese intra- und interindividuelle Variabilität von Mikrostrukturen wirft auch die Frage nach alters- und geschlechtsbedingten Unterschieden sowie nach Referenzwerten auf. Mit dieser Thematik beschäftigt sich der Beitrag von Danker-Hopfe et al. Auf der Basis von Daten aus der Siesta-Datenbank werden Geschlechtsunterschiede und Variationen mit dem Alter für 11 REM-Schlaf-bezogene Variablen untersucht. Darüber hinaus werden Referenzwerte - soweit erforderlich aufgeschlüsselt nach Geschlecht und Alter - berichtet.

Mit diesem Themenheft konnten lediglich ausgewählte Aspekte des Themas behandelt werden. Wir hoffen, damit Ihr Interesse an Mikrostrukturen des Schlafs geweckt zu haben und freuen uns darauf, vielleicht weitere Beiträge zu diesem Thema in einem der nächsten Hefte der Zeitschrift Somnologie - Schlafforschung und Schlafmedizin lesen zu können.

Ihre H. Danker-Hopfe

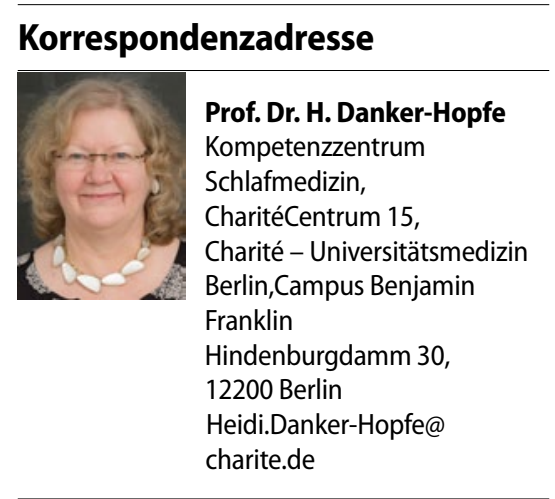

Interessenkonflikt. H. Danker-Hopfe gibt an, dass kein Interessenkonflikt besteht. 